\title{
$\begin{array}{llllllllll}E & D & \mid & T & O & R & \mid & A & L\end{array}$
}

\section{The north face of dento-facial orthopedics}

The field of dento-facial orthopedics now has at its disposal an ensemble of techniques based on the use of effectively perf forming mechanics to remediate the esthet. ic and functional consequences of malocclusions. In this limited frameworks, a morphological diagnosis is limited to the dental arches and the facial sheleton. Structural analysis continues to be only two-dimensional as does the soft tissues one, with an appreciation of the morphology of the nose being left to practitioners of other disciplines. Even though the number of specialties who share the responsisbility of providing treatment of problems in the cophalic region is impressively high, not one undertakes the task of integrating the examination of the morphology and pathology of its diverse domains. How can this compartmentalization possiblly do justice to the entivety of the facial complex?

The management of malocclusions has become so technical that it has persuaded some practitioners to view the two levels of the face as separate entities. Orthodontists sarely consider the intimate intertwining of dental malocclusions with disorders of nasal breathing, which they believe to be the exclusive province of ENJ specialists. Maxillfacial surgeons and some plastic surgeons, however, who are virtually the only ones to evaluate how the facial envelope integrates the changes they make in the face affect the underlying sheletal structures, constitute 
an exception. They fully understand that they need to recruit a sufficiently broad multidisciplinary team possessing a wide range of technical competencies to treat their patients adequately. Just because they are armed with remarkably efficient therapentic tools have orthodontists have any less need to comprehend the nature of malocclusions with an equivalent intellectual awareness of all aspects of the dento-facial complex?

Leonardo da Vinci said," Those who fall in love with practice without science are like a helmsman who sets sail without a tiller or a compass and never knows exactly where he is going. "Let us recognize that a proper diagnosis of difficulty in breathing at rest, shown by many patients, is far more complicated than we might think! Do we realize:

- that the specific role of the nasal cavities in breathing is to optimize the thermal exchanges needed for conditioning inspiratory air?

- that these exchanges are as dependant on the secretory response of the nasal epithelium as they are on nasal resistance?

- that in warming inspiratory air, the evaporation of the liquid from nasal secretion cools the turbinal venous blood that flows into the cavernous sinuses (placed on each side of the body of the sphenoid bone)?

- that this mechanism cools the blood of the internal carotid arteries passing through these sinuses and contributes to the thermoregulation of the deep centers of the cerebral hemispheres their convexity being cooled by the evaporation of sweat on the scalp)? 
- that their nasal thermoregulatory capacities of the moment aim at optimizing their breathing at rest, the others parts of their airways being fit to humidify inspiratory air?

- that the postural adaptations of the soft tissues encircling the mouth and nares openings control the mode of breathing at rest, conjointly, permanently, and completely?

- that in consequence the oro-nasal morphology of all patients results from the optimization of their breathing at rest by the posture they bestow on the oral and nasal regions of their facial envelopes (that practitioners observing in a narrow, specialized view tend to consider separately)?

- that the support the piriform aperture and the anterior portion of the maxillary dento-alveolar arch provide to the nasal and labial soft tissues of the facial envelope submits the morphogenesis of these structures to the postural consequences of any malfunction of nasal breathing?

- that correction of oral-nasal deformities by orthopedic expansion of the premaxilla and correction of associated nasal breathing malfunction by medical or surgical treatment, can contribute to the optimization of the maxillary arch form, of nasal breathing, of selective brain cooling, and of the posture of the cervico-cephalic soft tissues?

- that improvements in sleeping, of daily behavior, and of postures with the relief of obstructive malfunction that follow these corrections, all testify clinically to the optimization of heat transfer between inspiratory air and the nasal mucosa, which is only possible 
when the secretory capacity of the nasal mucosa and the extent of nasal resistance permit it?

Applied to the treatment of a congenitally absent maxillary lateral incisor, these data radically change an analysis of the results obtained. Formerly, the orthodontists technical arsenal provided him with two major options: open up or close the space between the canine and the central maxillary incisor. Cach of these options required supplementary procedures for completion of treatment of the arch. The results were rarely entirely satisfactory in view:

- of the neglected deformities in oral and nasal structure shapes, (characterized by insufficient transverse development, predominantly anterior, of the nasal cavities, of the piriform aperture, of the incisor arches, and of the labio-nasal and the labio-mental regions of the facial envelope), associated with malfunction of the nasal valves when the space was closed;

- or of the necessity to fill the re-opened space prosthetically. In these cases the optimization of breathing at rest and its bronchopulmonary and brain benefits that bestows add generously to the more obvious esthetic and functional gains.

Accordingly, in assessing a malocclusion it is important that orthodontists identify the nasal malfunction associated with a transverse premaxillary deficit, and its thermal ramifications. This enlarges the therapentic objective to include enhancement of development of the face, which orthodontists can accomplish only if they have a profound 
understanding of the functional targets of nasal breathing as the only way of finding a path to that goal. Orthodontists are realizing that more and more malocclusions can be considered as the alveolodental components of more extensive oro-nasal deformities that develop within the framework of the morphological and pathological integration that operates continuously in the facial envelope.

Finally,

- because the joint role that the oral and nasal openings of the facial envelope play in the determination of an individual's breathing mode is permanent and vital;

- because the targets of nasal breathing at rest are limited neither to the pulmonary alveoli nor to the olfactory receptors;

the capability of contributing at an opportune time to a great improvement in oro-nasal function and cervico-cephalic posture thanks to its therapeutic modeling of certain facial forms opens up for dentofacial orthopedics vast new treatment perspectives that stretch far beyond the esthetic objectives currently allocated to it. When the discernment of orthodontists has led them to integrate in their treatment all the components of oro-facial deformities, these one-time technicians will have become true therapists: they will have climbed the Morth Face of the Mount Everest of orthodontics.

With all the respect that face deserves.

Jacques TALMANT 